\title{
Label-free and washing-free alkaline phosphatase assay using a personal glucose meter
}

\author{
Jun Ki Ahn ${ }^{1 \dagger}$, Hyo Yong Kim ${ }^{1 \dagger}$, Chang Yeol Lee ${ }^{1}$, Ki Soo Park² ${ }^{*}$ and Hyun Gyu Park ${ }^{1 *}$
}

\begin{abstract}
We herein describe a personal glucose meter (PGM)-based method for a label-free and washing-free determination of alkaline phosphatase (ALP) activity, which relies on the cascade enzymatic reactions promoted by hexokinase and pyruvate kinase to couple ALP activity with the amount of glucose. In principle, the presence of target ALP scavenges on adenosine 5'-triphosphate (ATP), a phosphate source for hexokinasecatalyzed reactions, and thus suppresses the ensuing cascade enzymatic reactions. As a result, the initial high amount of glucose is maintained and the amount of glucose, which is proportional to ALP activity, is simply measured by a hand-held PGM. Based on this novel strategy, we successfully determined the ALP activity down to $8.9 \mathrm{U} / \mathrm{L}$ with the high selectivity. In addition, the diagnostic capability of this method was demonstrated by reliably assaying the ALP activity in non-diluted human blood without any pretreatment steps.
\end{abstract}

Keywords: Alkaline phosphatase, Adenosine 5'-triphosphate, Personal glucose meter, Cascade enzymatic reaction, Biosensor

\section{Background}

A phosphatase is an enzyme that removes phosphate groups from its substrate by catalyzing the hydrolysis of a phosphomonoester and produces the molecule containing free hydroxyl groups. This enzyme is universally distributed in the cellular membrane and plays a critical role in the regulation of intracellular processes involved in cell cycle, growth, apoptosis, and signal transduction [1]. The most representative example in this type of enzyme is alkaline phosphatase (ALP), which is found in all tissues throughout the body, but is more concentrated on liver, bones, placenta, intestines, and kidneys [2]. Its abnormal

\footnotetext{
* Correspondence: kskonkuk@gmail.com; hgpark@kaist.ac.kr

†Jun Ki Ahn and Hyo Yong Kim contributed equally to this work.

Jun Ki Ahn and Hyo Yong Kim are co-first author.

${ }^{2}$ Department of Biological Engineering, College of Engineering, Konkuk

University, Seoul 05029, Republic of Korea

'Department of Chemical and Biomolecular Engineering (BK21+ Program),

KAIST, 291 Daehak-ro, Yuseong-gu, Daejeon 34141, Republic of Korea
}

level in serum is found to be closely related to various diseases and thus it has been used as a biomarker for the diagnosis of various diseases [3]. For examples, the low level of ALP in serum is often observed in malnourished patients, while the high level of ALP in serum is commonly associated with liver dysfunction, bone disease, prostate cancer, and bile-duct obstruction [4].

Up to date, various ALP assays have been developed, which rely on chromatographic, chemiluminescent, fluorescent, electrochemical, and surface enhanced Raman scattering signaling methods. However, most of them are not practically utilized due to their drawbacks such as the requirement for expensive reagents and specialized equipment. The ELISA method, which relies on the ALP-catalyzed conversion of $p$-nitrophenyl phosphate ( $p$ NPP) onto yellow colored products, is only used in the clinics to monitor

(c) The Author(s). 2019 Open Access This article is distributed under the terms of the Creative Commons Attribution 4.0 International License (http://creativecommons.org/licenses/by/4.0/), which permits unrestricted use, distribution, and 
ALP activity. A number of ELISA kits are commercially available on the market, but this method still has limitations such as the involvement of complicated operation, long assay time, and expensive equipment. Therefore, the development of novel strategies that operate in a stable, convenient, and cost-effective manner is highly required to replace ELISA-based ALP detection.

Towards this goal, we herein developed a simple method for a label-free and washing-free determination of ALP activity by employing a personal glucose meter (PGM), which has been regarded as one of the most successful point-of-care (POC) devices with a number of unique features including high portability, low cost, simple operation, and reliable quantitative ability [5]. Based on our recent finding that ATP was reliably determined in various real samples by linking the amount of ATP to glucose that is detectable by PGM, we designed a new PGM-based strategy for the determination of ALP activity [6]. With the developed system, we successfully analyzed the target ALP by simply measuring the PGM signal that is dependent on ALP activity, while overcoming the drawbacks in the conventional ALP assays.

\section{Methods}

\section{Materials}

D-glucose, magnesium chloride $\left(\mathrm{MgCl}_{2}\right)$, Tris(hydroxymethyl)aminomethane hydrochloride (Tris- $\mathrm{HCl}), \quad \beta$ nicotinamide adenine dinucleotide phosphate hydrate ( $\beta$-NADP), phosphoenolpyruvic acid (PEP), adenosine 5 '-triphosphate disodium salt hydrate (ATP), alkaline phosphatase (ALP), human serum albumin (HSA), bovine serum albumin (BSA), trypsin, lysozyme, avidin, thrombin, hexokinase, glucose-6-phosphate dehydrogenase (G6PD), and pyruvate kinase were purchased from Sigma-Aldrich (St. Louis, MO, USA). Human blood was purchased from ZenBio Inc. (Research Triangle Park, NC, USA). All other chemicals were of analytical grade and used without further purification. Aqueous solutions were prepared using ultrapure DNase/RNase-free distilled water (D.W.) purchased from Bioneer. The personal glucose meter (PGM) from Accu-Chek Active (Roche, Basel, Switzerland) was used in this work.

\section{Alkaline phosphatase assay using a PGM}

$16 \mu \mathrm{L}$ of ALP at various concentrations or HSA, BSA, trypsin, lysozyme, avidin, and thrombin $(2.5 \mathrm{uM})$ was first mixed with $2 \mu \mathrm{L}$ of ATP $(50 \mathrm{mM})$ and $2 \mu \mathrm{L}$ of $10 \mathrm{X}$ reaction buffer $(500 \mathrm{mM}$ Tris- $\mathrm{HCl}, 10 \mathrm{mM}$ $\mathrm{MgCl}_{2}, \mathrm{pH} 7.4$ ) and incubated at $37^{\circ} \mathrm{C}$ for $30 \mathrm{~min}$. Next, the above ALP reaction solution was mixed with $5 \mu \mathrm{L}$ of D-glucose $(50 \mathrm{mM}), 1 \mu \mathrm{L}$ of $\beta$-NADP (50
$\mathrm{mM}), 1 \mu \mathrm{L}$ of PEP $(100 \mathrm{mM})$, and $5 \mu \mathrm{L}$ of $10 \mathrm{X}$ reaction buffer ( $1 \mathrm{M}$ Tris- $\mathrm{HCl}, 100 \mathrm{mM} \mathrm{MgCl}_{2}, \mathrm{pH} 7.4$ ), which was then incubated with $18 \mu \mathrm{L}$ of enzyme mixture containing hexokinase $(5 \mathrm{U})$, pyruvate kinase (5 $\mathrm{U})$, and G6PD $(0.4 \mathrm{U})$ at $30^{\circ} \mathrm{C}$ for $30 \mathrm{~min}$. Finally, the resulting glucose level in the mixture was measured by a PGM.

\section{Recovery test}

The target ALP at different concentrations were spiked into non-diluted human blood, which was directly analyzed by following the same detection procedure used in the buffer solution. To determine the amount of spiked ALP, the calibration curve was first drawn with a set of standards containing a known amount of ALP in the non-diluted human blood and the unknown amount of ALP was then determined based on the calibration curve.

\section{Results and discussion \\ Detection principle of the PGM-based ALP assay}

The conceptual design of the PGM-based ALP assay is illustrated in Fig. 1. The proposed system relies on the cascade enzymatic reactions promoted by hexokinase and pyruvate kinase that link the amount of ATP to glucose [6]. In the absence of target ALP, the intact ATP enables hexokinase to catalyze the conversion of glucose to glucose-6-phosphate by providing a phosphate group to glucose and accordingly the amount of glucose is decreased. In addition, the adenosine $5^{\prime}$-diphosphate (ADP), which is generated after the hexokinase-catalyzed enzymatic reaction, is recovered to ATP by pyruvate kinase that catalyzes the conversion of phosphoenolpyruvic acid (PEP) to pyruvate. The regenerated ATP is again supplemented to catalyze multiple rounds of cascade enzymatic reactions, resulting in a significantly decreased amount of glucose. On the other hand, the presence of ALP scavenges on ATP, which suppresses the subsequent, cascade enzymatic reactions promoted by hexokinase and pyruvate kinase. Notably, ALP shows the best enzymatic activity at $\mathrm{pH}$ values from 8 to 10 , at which other enzymes such as hexokinase, pyruvate kinase, and G6PD can hardly resist. Thus, we selected $\mathrm{pH} 7.4$ at which other enzymes work fine and ALP activity for the hydrolysis of ATP is slightly reduced to ca. $80 \%$ [7]. Under these conditions, we can successfully monitor the ALP activity using a PGM despite the slight sacrifice of ALP activity. As a result, the initial high amount of glucose is retained and the amount of glucose that is proportional to ALP activity is simply monitored by a hand-held PGM. 


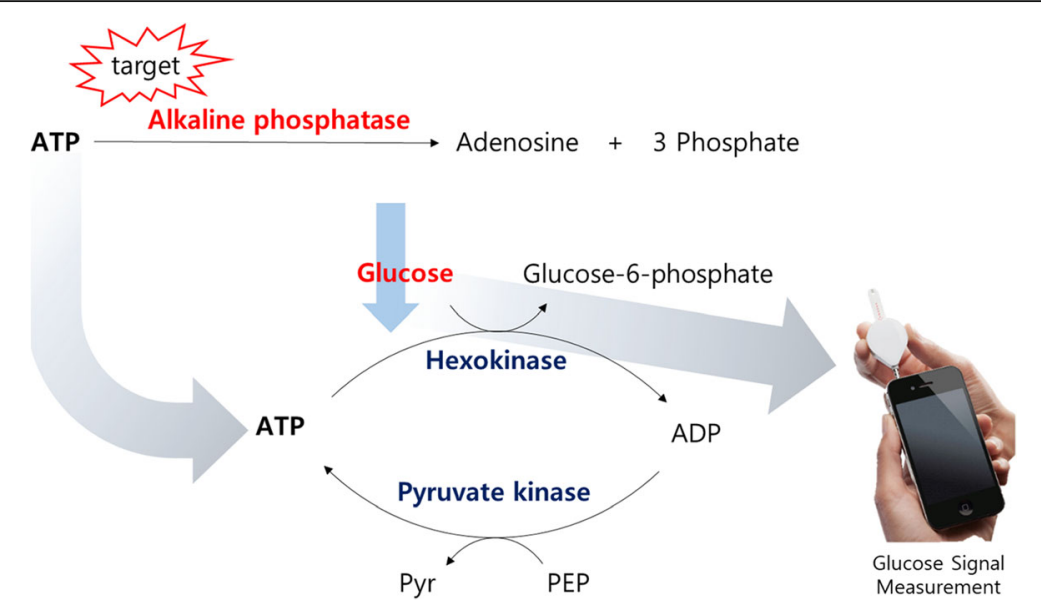

Fig. 1 Schematic illustration of the PGM-based ALP assay (ATP: adenosine 5'-triphosphate, ADP: adenosine 5'-diphosphate, PEP: phosphoenolpyruvic acid, Pyr: Pyruvate)

\section{Detection feasibility of the PGM-based ALP assay}

First, the detection feasibility of the PGM-based ALP assay was validated by measuring PGM signals in different samples. As shown in Fig. 2, the significantly reduced PGM signal was observed in the absence of target ALP because the intact ATP initiated the cascade enzymatic reactions promoted by hexokinase and pyruvate kinase, resulting in the decrease of glucose amount (Fig. 2(a)). However, in the presence of target ALP, the ATP was wasted and the subsequent cascade

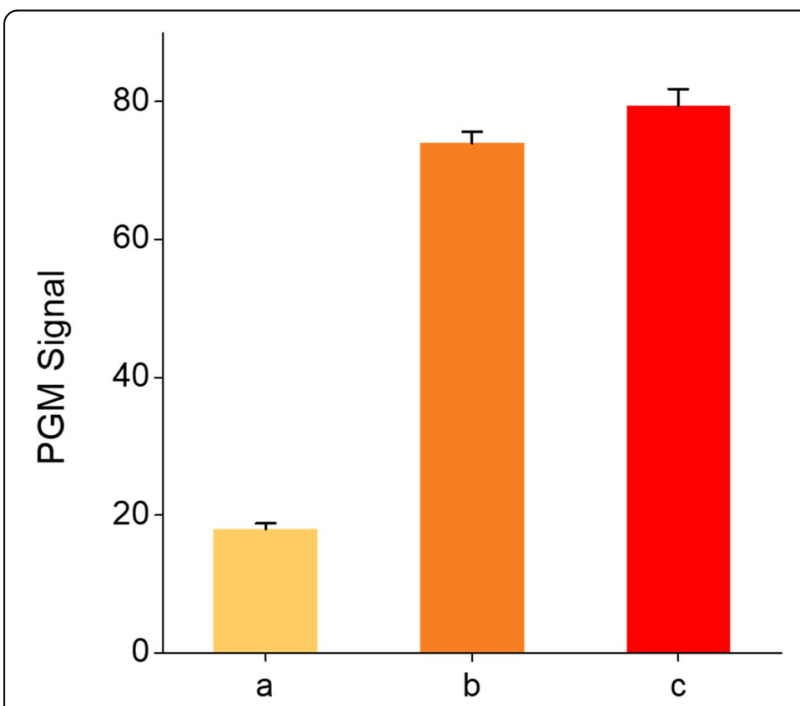

Fig. 2 Detection feasibility of the PGM-based ALP assay. The PGM signals from different samples containing ATP only (a), both ALP and ATP (b), and neither ALP nor ATP (c). The concentrations of ALP and ATP were $400 \mathrm{U} / \mathrm{L}$ and $1 \mathrm{mM}$, respectively enzymatic reactions were not executed, and thus the high PGM signal was obtained (Fig. 2(b)), which was comparable to the one of the negative control in which both ALP and ATP were excluded (Fig. 2(c)). These observations clearly confirm that ALP that utilizes ATP as the substrate regulates the cascade enzymatic reactions and the resulting changes of glucose amount are simply monitored by a hand-held PGM. Next, the optimal conditions for the efficient analysis of ALP activity were also investigated. The results of experiments in which the ATP amount and ALP reaction time were varied demonstrate that 5 $\mathrm{mM}$ of ATP and $30 \mathrm{~min}$ of ALP reaction were ideal to achieve the best detection performance, which were used for further experiments (Additional file 1: Figure S1).

\section{Selectivity of the PGM-based ALP assay}

The selectivity of the PGM-based ALP assay was investigated by examining the abilities of interfering proteins such as BSA, HSA, trypsin, lysozyme, avidin, and thrombin to change the PGM signal. As presented in Fig. 3, the high PGM signal was only observed from the sample containing the ALP. On the other hand, the presence of interfering proteins produced the negligible PGM signal even though their concentrations were at four times higher than that of ALP. These results clearly confirm the high selectivity of our ALP detection method.

\section{Sensitivity of the PGM-based ALP assay}

The sensitivity of the PGM-based ALP assay was determined by measuring the PGM signal as a function of ALP concentration. The results in Fig. 4 show 


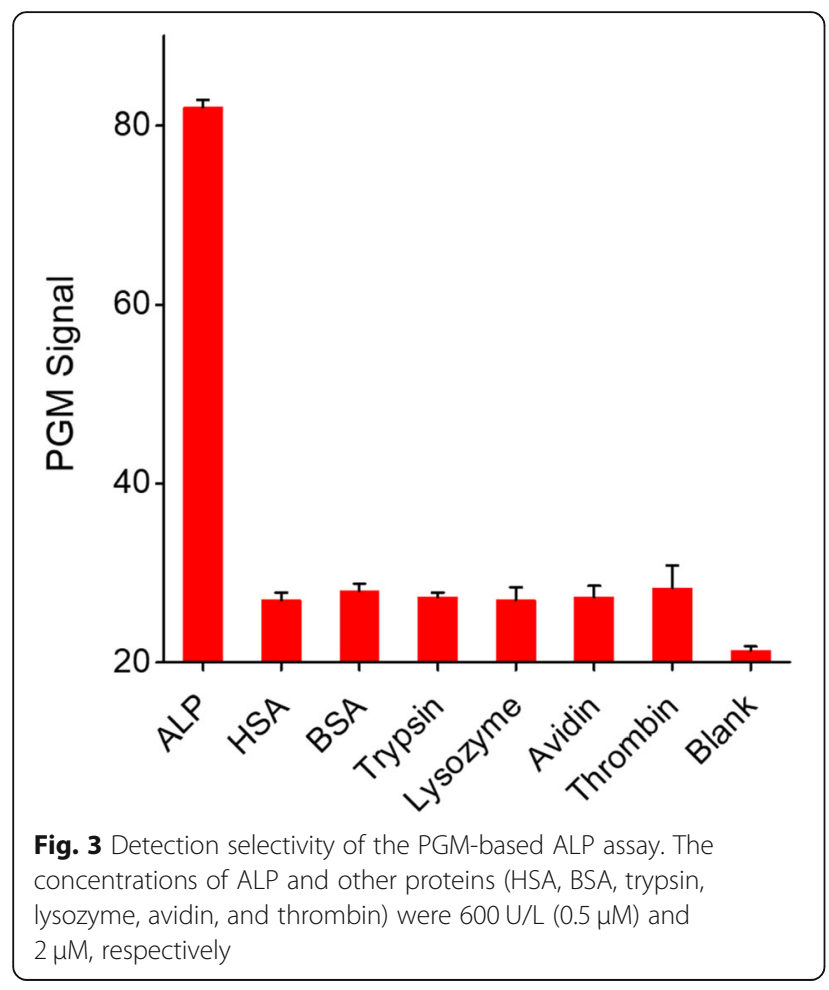

that the PGM signal increases with increasing concentration of ALP. An excellent linear relationship $\left(R^{2}=0.9937\right)$ existed in the range from 8 to $200 \mathrm{U} / \mathrm{L}$

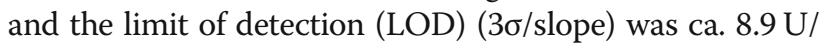
$\mathrm{L}$ (7.4 pM), which is comparable or higher than those from other ALP assay methods (Additional file 1:

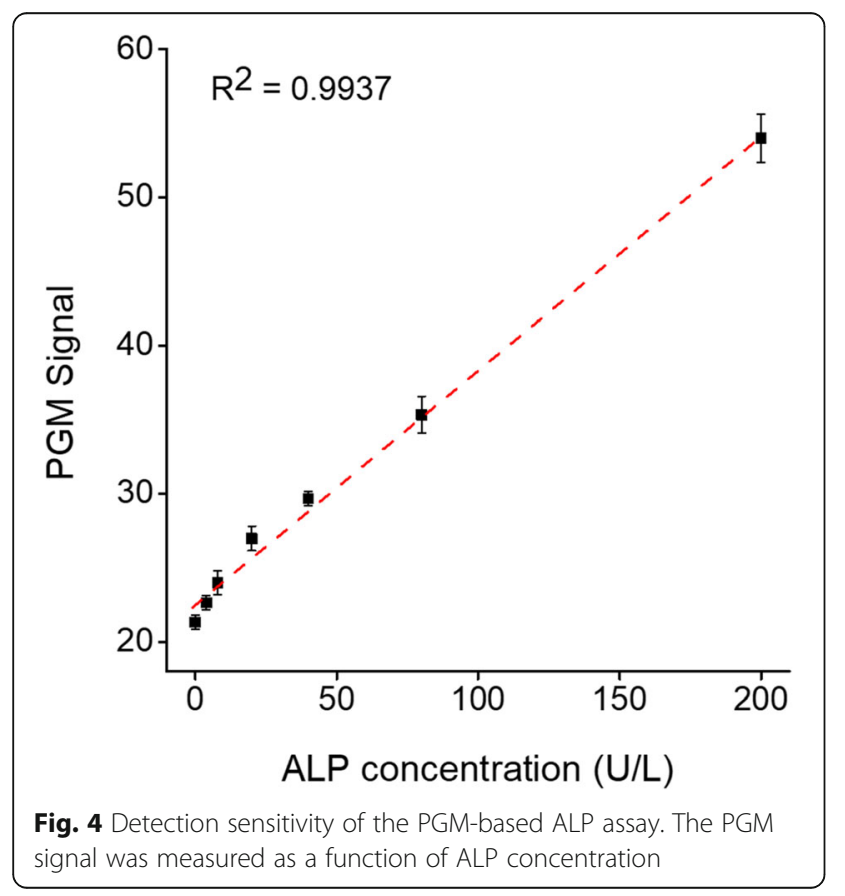

Table S1). However, it should be noted that the detection sensitivity of our strategy is good enough to cover the clinically important concentration of ALP in human blood (44-147 U/L) [8]. In addition, the developed method is simply operated by a hand-held PGM without the requirement of bulky operation system and expensive reagents.

\section{Practical applicability of the PGM-based ALP assay}

Finally, the practical applicability of the PGM-based ALP assay was demonstrated by analyzing ALP present in non-diluted human blood. As shown in Fig. 5, ALP activity in the human blood was determined based on the standard addition method [9]. The results demonstrate that the PGM signal increased with increasing concentration of ALP spiked into human blood in the range from 0 to $200 \mathrm{U} / \mathrm{L}$ and the ALP activity present in the human blood was determined to be $66 \mathrm{U} / \mathrm{L}$ which is in good agreement with the typical ALP activity in the human blood [8]. Furthermore, the excellent reproducibility and precision of our strategy was confirmed by a coefficient of variation $(\mathrm{CV})$ less than $9 \%$ and a recovery ratio between 98 and 107\% (Table 1). Importantly, the ALP in human blood was analyzed without either dilution or pre-treatment steps, which is normally executed in most ALP methods to avoid the signal interference [10]. Overall, these results prove that the developed ALP system could be utilized to reliably determine the ALP activity in human blood and applied to the assay of ALP contained in the whole blood samples obtained by finger prick, ensuring its future clinical application.

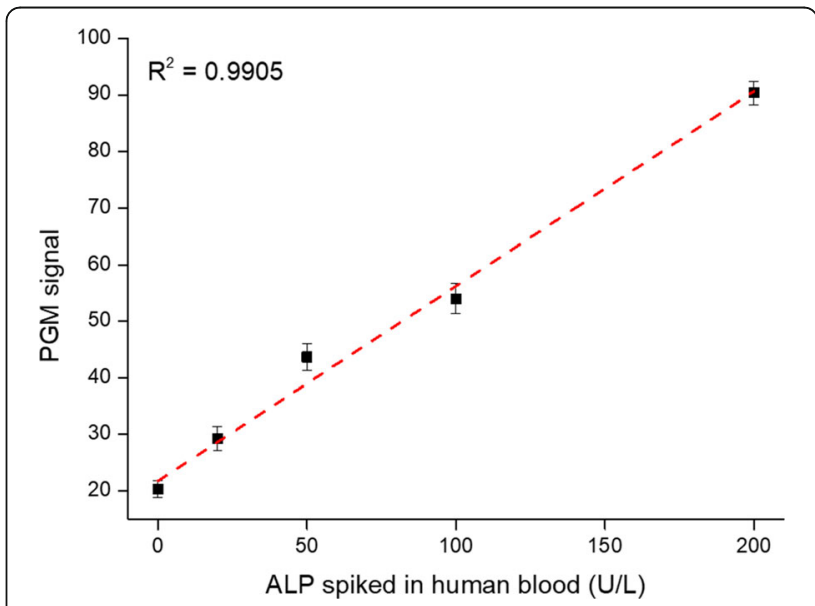

Fig. 5 The PGM signal as a function of ALP spiked in non-diluted human blood 
Table 1 Determination of ALP spiked into non-diluted human blood ${ }^{a}$

\begin{tabular}{llllll}
\hline ALP in human blood $(\mathrm{U} / \mathrm{L})$ & Added ALP $(\mathrm{U} / \mathrm{L})$ & Measured ALP $(\mathrm{U} / \mathrm{L})^{\mathrm{b}}$ & $\mathrm{SD}^{\mathrm{c}}$ & $\mathrm{CV}_{(\%)^{\mathrm{d}}}$ & Recovery $(\%)^{\mathrm{e}}$ \\
\hline 66 & 30 & 101.94 & 8.85 & 8.68 & 106.19 \\
& 50 & 113.74 & 5.11 & 4.49 & 98.05 \\
& 100 & 168.82 & 9.01 & 5.34 & 101.70 \\
\hline
\end{tabular}

${ }^{a}$ To determine the concentration of ALP in human blood, a calibration curve was first created by using standards having known concentrations of ALP in human blood (Fig. 5). Based on this calibration curve, the PGM signals from unknown samples were used to determine the concentrations of ALP in human blood. ${ }^{b}$ Mean of three measurements. ${ }^{C}$ Standard deviation of three measurements. ${ }^{d}$ Coefficient of variation $=\mathrm{SD} / \mathrm{mean} \times 100$. ${ }^{\mathrm{e}}$ Measured value/added value $\times 100$

\section{Conclusions}

We herein developed a PGM-based method for a label-free and washing-free ALP assay. This strategy relies on the working principle that ALP, which scavenges on ATP and suppresses the subsequent cascade enzymatic reactions, changes the amount of glucose that is simply measured by a hand-held PGM. With this design principle, the target ALP was successfully determined with high selectivity even in human blood. Importantly, the developed system was simply operated with a PGM, while overcoming the drawbacks in the previous ALP assays that require expensive reagents and specialized equipment. In addition, the developed ALP assay utilizes a PGM that is commercially available on the market at the low cost and thus it would be readily applied in a various POC tests. Finally, we believe that the developed system would be practically used in the clinics and pave the way for the new enzymatic assay.

\section{Additional file}

Additional file 1: Table S1. Summary of the previously reported ALP assay methods. Figure S1. Optimization of (a) ATP concentration and (b) ALP reaction time. $P_{0}$ and $P$ are defined as $P G M$ signals in the absence and presence of ALP (200 U/L), respectively. (DOCX $191 \mathrm{~kb}$ )

\section{Abbreviations}

ALP: alkaline phosphatase; ATP: adenosine 5'-triphosphate; BSA: bovine serum albumin; G6PD: glucose-6-phosphate dehydrogenase; HSA: human serum albumin; PEP: phosphoenolpyruvic acid; PGM: personal glucose meter; Tris-HCl: Tris(hydroxymethyl)aminomethane hydrochloride; $\beta$-NADP: $\beta$ nicotinamide adenine dinucleotide phosphate hydrate

\section{Acknowledgements}

Not applicable.

\section{Authors' contributions}

JKA originally developed the main idea. JKA and HYK mainly performed the research by designing experiment and acquiring data. CYL supported the research by discussing about the experimental results. KSP and HGP, as corresponding authors, led the research by suggesting the helpful guidance for the progress of the research and finally revised and approved the manuscript.

\section{Funding}

This research was supported by BioNano Health-Guard Research Center funded by the Ministry of Science and ICT (MSIT) of Korea as Global Frontier Project (Grant number H-GUARD_2013M3A6B2078964). This research was also supported by the Mid-career Researcher Support Program of the
National Research Foundation (NRF) funded by the Ministry of Science, ICT \& Future planning (MSIP) of Korea (NRF-2018R1A2A1A05022355).

Availability of data and materials

All data generated or analyzed during this study are included in this published article [and its supplementary information files].

Ethics approval and consent to participate

Not applicable.

\section{Consent for publication}

Not abblicable.

\section{Competing interests}

The authors declare that they have no competing interests.

Received: 25 February 2019 Accepted: 27 May 2019

Published online: 04 June 2019

\section{References}

1. Julien SG, Dubé N, Hardy S, Tremblay ML. Inside the human cancer tyrosine phosphatome. Nat Rev Cancer. 2011;11:35-49.

2. Hartwell SK, Somprayoon D, Kongtawelert P, Ongchai S, Arppornchayanon O, Ganranoo L, et al. Online assay of bone specific alkaline phosphatase with a flow injection-bead injection system. Anal Chim Acta. 2007;600:188-93.

3. Fanjul-Bolado P, Hernández-Santos D, González-García MB, Costa-García A. Alkaline phosphatase-catalyzed silver deposition for electrochemical detection. Anal Chem. 2007;79:5272-7.

4. Jiang $H$, Wang $X$. Alkaline phosphatase-responsive anodic electrochemiluminescence of CdSe nanoparticles. Anal Chem. 2012;84: 6986-93.

5. Carroll AE, Marrero DG, Downs SM. The HealthPia GlucoPack ${ }^{\mathrm{TM}}$ diabetes phone: a usability study. Diabetes Technol Ther. 2007;9:158-64.

6. Ahn JK, Kim HY, Park KS, Park HG. A personal glucose meter for label-free and washing-free biomolecular detection. Anal Chem. 2018;90:11340-3.

7. Demenis MA, Leone FA. Kinetic characteristics of ATP hydrolysis by a detergent-solubilized alkaline phosphatase from rat osseous plate. IUBMB Life. 2000:49:113-9.

8. Hadizadeh M, Abedi SH, Malekpour H, Radinnia E, Jabbehdari S, Padashi M, et al. Prevalence of inflammatory bowel disease among patients with primary sclerosing cholangitis in Iran. Arab J Gastroenterol. 2016;17:17-9.

9. Park KS, Kim MI, Woo MA, Park HG. A label-free method for detecting biological thiols based on blocking of $\mathrm{Hg}^{2+}$-quenching of fluorescent gold nanoclusters. Biosens Bioelectron. 2013;45:65-9.

10. Qian ZS, Chai LJ, Huang YY, Tang C, Jia Shen J, Chen JR, et al. A real-time fluorescent assay for the detection of alkaline phosphatase activity based on carbon quantum dots. Biosens Bioelectron. 2015;68:675-80.

\section{Publisher's Note}

Springer Nature remains neutral with regard to jurisdictional claims in published maps and institutional affiliations. 